\title{
Downregulation of SMC4 Inhibits the Progression of Cardia Adenocarcinoma via PI3K/ AKT Signaling Pathway Regulation
}

\section{Mengqi Zhu}

First Affiliated Hospital of Bengbu Medical College

\section{Xinxin Zhang}

First Affiliated Hospital of Bengbu Medical College

Kaiji Gao

First Affiliated Hospital of Bengbu Medical College

\section{Lingmei Zhang}

First Affiliated Hospital of Bengbu Medical College

\section{Xiaojia Feng}

First Affiliated Hospital of Bengbu Medical College

\section{Hui Wang}

First Affiliated Hospital of Bengbu Medical College

Jianguang Jia ( $\nabla$ jiajianguang1978@126.com )

First Affiliated Hospital of Bengbu Medical College https://orcid.org/0000-0001-8644-3256

\section{Research}

Keywords: SMC4, cardiac adenocarcinoma, PI3K/ AKT signaling pathway

Posted Date: July 12th, 2021

DOI: https://doi.org/10.21203/rs.3.rs-698892/v1

License: (c) (1) This work is licensed under a Creative Commons Attribution 4.0 International License. Read Full License 


\section{Abstract}

Background: Cardia adenocarcinoma (CA) is a subtype of gastric cancer with a high rate of local and distal recurrence and few targeted therapies. Structural maintenance of chromosome protein 4 (SMC4) is involved in the occurrence and progression of numerous malignancies, but its role and mechanism in CA are unknown.

Methods: Through the Western Blot and qRT-PCR, the level of SMC4 expression was determined in CA. SMC4 knockout cells were then generated by stable transduction of BGC-823 and SGC-7901 cells. Cell proliferation was evaluated by MTT and clone formation test, Scratch and transwell tests were used to investigate cell migration as well as invasion, while through the flow cytometry, we examined the cell apoptosis and progression of the cell cycle. The regulatory effects of the epithelial-mesenchymal transition (EMT) and the PI3K/AKT pathway were investigated using Western Blot.

Results: This study showed overexpression of SMC4 in various CA cells. SMC4 knockout significantly caused the inhibition of proliferation, migration, and invasion of BGC-823 and SGC-7901, and stimulate the process of apoptosis and cell cycle arrest in the G0/G1 phase. In addition, down-regulation of SMC4 resulted in decreased expression of Bcl-2, Cyclin D1, CDK4, CDK6, N-cadherin and Vimentin, with an increased level of proteins i.e Bax, caspase3, Cleaved-caspase3, P21, and E-cadherin. SMC4 knockout also reduced the phosphorylated protein levels of AKT, PI3K, and mTOR, while keeping their total protein levels constant.

Conclusion: Downregulation of SMC4 can inhibit the biological progression of CA, suggesting that SMC4 could be a potential therapeutic target for the disease.

\section{Background}

One of the most prevalent malignant tumors of the digestive tract is gastric cancer (GC), and its morbidity and mortality account for a considerable proportion of all malignant tumors worldwide [1]. Although the GC mortality and incidence in China have been on a downward trend, the increasing and aging population still leads to a substantial increase in the number of new cases every year [2]. Cardia adenocarcinoma (CA) is a type of GC, and its tumor center is located within $5 \mathrm{~cm}$ of the proximal and distal anatomical Cardia $[3,4]$. CA has been on the rise in Asian countries in recent years, with China having a much greater prevalence of Siewert type II and type III tumors, while the prevalence of type I tumors is relatively low [5]. Surgery is still the most common treatment for $\mathrm{CA}$, however, due to the high rates of local and distal recurrence, surgical treatment alone is usually ineffective [6]. Targeted therapy includes trastuzumab, ramoxicumab and pemumumab, which have good therapeutic results for patients with advanced or metastatic CA [7], suggesting that targeted therapy is feasible to some extent. The survival rate of patients can be enhanced if cancer can be discovered early. However, it is frequently advanced by the time it is diagnosed. Therefore, the survival rate of patients with CA is still not high. More therapeutic 
targets and biomarkers must be recognized for CA patients to obtain early diagnosis and improved therapy, hence improving patients' overall survival rates.

Structural maintenance of chromosome protein 4 (SMC4) is a protein that belongs to the SMC family. Located in the 3 q25. 33 (https://www.ncbi.nlm.nih.gov/gene/10051) [8]. SMC4, the core subunit of condensation proteins I and II, is involved in chromosomal aggregation and mitotic sister chromatid separation, which has been linked to the regulation of cell cycle progression in various malignancies, as well as tumor growth and carcinogenesis $[9,10]$. In recent years, there have been increasing reports on the SMC4 gene in colorectal cancer [11], liver cancer [10], lung adenocarcinoma [12], breast cancer [8], acute myeloid leukemia [9], and glioma [13] and other tumors. According to research findings, the SMC4 gene has a vital role in the occurrence and progression of known cancers, such as lung adenocarcinoma and glioma. However, its expression, potential role, and mechanism in CA are still unclear.

In this work, we found that SMC4 expression was increased in CA cell lines. Downregulation of SMC4 can inhibit cell proliferation, invasion, and migration ability, block cell cycle progression, promote cell apoptosis, and inhibit the EMT process. In addition, these regulatory effects of SMC4 on CA are likely to be realized through the regulation of the PI3K/ AKT signaling pathway. Therefore, targeting the SMC4 gene could be an effective approach to inhibit the progression of $\mathrm{CA}$, and these results may provide a new target for CA treatment and research.

\section{Materials And Methods}

\subsection{Cell lines and cell culture}

Human gastric cancer cell lines BGC-823, SGC-7901, MGC-803, and MKN-45 were all purchased from Shanghai Cell Bank, Chinese Academy of Sciences (Shanghai, China). Wuhan Procell Life Science \& Technology Co., Ltd (Wuhan, China) provided the human gastric mucosal epithelial cell GES-1. These cells were grown at $37^{\circ} \mathrm{C}$ in a humidified environment of $5 \% \mathrm{CO}_{2}$ in RPMI-1640 media (Gibco, USA) supplemented with fetal bovine serum (10\%, Gibco, USA) and streptomycin-penicillin (1\%). The medium was changed every two days.

\subsection{Lentivirus Infection}

In order to knock out SMC4, we purchased two sequences of shRNA (RNAi1:GGGUAUGCUUGAAUAUUUATTUAAAUAUUCAAGCAUACCCTT; RNAi2:GCCCAAGAAUGUGUAAACUTTAGUUUACACAUUCUUGGGCTT) from Invitrogen (CA, USA) followed by their cloning into the pSUPER-puro plasmid (GeneChem, Shanghai, China) to generate SMC4-RNAi constructs. As mentioned earlier, lentivirus particle production and infection are performed. Then purinomycin was used to screen out cells that stably knocked out the SMC4 gene.

\section{3 qRT-PCR}


Extraction of total RNA was performed through using a Trizol reagent (Invitrogen, USA) as directed by the manufacture, and the quality of total RNA was tested in a 260/280 ratio using a NanoVue ${ }^{\text {TM }}$ Plus (Thermo Fisher Scientific, MA, USA) instrument. Then, using PrimeScript ${ }^{\mathrm{TM}}$ 1st Strand cDNA Synthesis kit (Takara, Kusatsu, Japan) to synthesize cDNA. Quantitative reverse transcription-polymerase chain reaction was performed using ABI StepOne ${ }^{\text {TM }}$ Real-Time PCR System (Applied Biosystems, CA, USA) and SYBR Premix $\mathrm{Ex} \mathrm{Taq}^{\mathrm{TM}}$ (TaKaRa). GAPDH was used as an endogenous reference.

The primer sequences used in qRT-PCR are as follows: SMC4 forward: 5'-GAGAAAATTCTGGGACCTTT-3', SMC4 reverse: 5'-TCTGAATGTCCTTGTGTTCA-3'; GAPDH forward: 5'-ACCACAGTCCATGCCATCAC-3', GAPDH reverse: 5'-TCCACCACCCTGTTGCTGTA-3'.

\subsection{MTT assay}

Cells at a density of $7 \times 10^{3}$ with a stable knockout of the SMC4 gene were seeded in 96-well plates (Corning, NY, USA) and cultured in $5 \% \mathrm{CO}_{2}$ at $37^{\circ} \mathrm{C}$. MTT was added to each well on days $1,2,3,4$, and 5 , and incubated for $4 \mathrm{~h}$, then the supernatant was discarded followed by the incorporation of dimethylsulfoxide (DMSO) (Sigma, USA) for MTT assay to test cell viability. The optical density (OD) was measured at $490 \mathrm{~nm}$ while employing a microplate reader (Bio-Rad, CA, USA).

\subsection{Colony Formation Assay}

Cells that had been steadily knocked out of SMC4 were inoculated in a 6-well plate at $3 \times 10^{3}$ per hole density and incubated for 14 days at $37^{\circ} \mathrm{C}$ and $5 \% \mathrm{CO} 2$. The fixation of cells was then performed with 4 $\%$ polyformaldehyde, then dyed with crystal violet, dried, and the colonies of more than 50 cells were counted.

\subsection{Wound healing assay}

At a density of $6 \times 10^{5}$ cells per well, cells that had been stably knocked out of SMC4 were seeded into 6well plates. When they grew to more than $90 \%$, the cell surface was scraped with a sterile pipette tip and washed to remove cell debris. Imaging was performed under a microscope at 0 and 24 hours after treatment, respectively. The wound area was quantified using ImageJ and the healing rate was calculated to assess mobility after 24 hours.

\subsection{Assays for migration and invasion}

To detect cell movement and invasion, a transwell method was used. SMC4 knockout cells were suspended in the media free with serum and injected into the upper chamber at a density of $2 \times 10^{4}$ per well. Fetal bovine serum (10\%) containing medium was filled to the lower chamber and cultivated at $37^{\circ} \mathrm{C}$ in a humidified environment with $5 \% \mathrm{CO} 2$. After incubation for 24 hours, they were rinsed with PBS, and subjected for fixation with $4 \%$ paraformaldehyde and crystal violet staining, and counted under a microscope. The migration test was described above, while the invasion test was precoated with $50 \mathrm{ul}$ of matrigel at the bottom of the upper chamber and incubated for 48 h.using the same steps as done in the migration test. 


\subsection{Flow Cytometry Analysis}

For apoptosis, cells were typically digested with trypsin and washed. Annexin V-FITC Cell Apoptosis Detection Kit (YEASEN Biotech Co., Ltd., Shanghai, China) was employed for dying the cells as directed by the manufacturer. Finally, through a Flow Cytometer (BD Biosciences) we detected stained cells, and the apoptotic data was analyzed with FLOWJO software (Tree Star, Inc, Ashland, OR).

Harvested cells were rinsed in cold PBS and fixed in 70\% ethanol (pre-cooled) for at least 2 hours for the cell cycle. The cells were stained with Propidium iodide (Sigma, USA) after being rinsed again with precooled PBS. Similarly, the stained cells were perceived by Flow Cytometer and the effects of SMC4 on various stages of the cell cycle were analyzed by FLOWJO software.

\subsection{Western Blotting}

RIPA cell lysate (Solarbio, Beijing, China) with protease and phosphatase inhibitors were used for extracting the total protein while its concentration was quantified with a BCA protein detection kit (Beyotime Institute of Biotechnology, China). After that, the protein separation was performed on an SDSPAGE gel through the process of electrophoresis and shifted to PVDF membranes (Millipore, USA). Then we treated the membranes with primary antibody at $4^{\circ} \mathrm{C}$ overnight after 30 minutes of rapid closure. After 2 hours of incubation with appropriate secondary antibodies labeled with Horseradish Peroxidase (HRP), the visualization of protein bands was carried out using ECL (Beyotime) method.

Antibodies against SMC4, a-tublin, Bcl-2, Bax, caspase3, Cleaved-caspase3, CDK4, CDK6, Cyclin D1, P21, E-cadherin, Vimentin N-cadherin, (Proteintech, Wuhan, China), P-PI3K, PI3K, AKT, mTOR, P-AKT, P-mTOR (Abcam, Cambridge, UK) were mainly used in this paper. The internal reference for the expression levels of these proteins was a-tublin.

\subsection{Statistical analysis}

Through GraphPad Prism 7 (GraphPad Inc., La Jolla, CA, USA), all data were analyzed. The data was gathered from three separate experiments and shown as mean \pm SEM. ANOVA was used to compare the difference analysis, and $\mathrm{P}<0.05$ was considered to be statistically significant.

\section{Results}

\subsection{SMC4 is overexpressed in CA}

Western Blot showed the upregulation of SMC4 in all CA cell lines compared with GES-1 (Fig. 1A). qRTPCR assay showed that SMC4 was overexpressed in the above multiple CA cell lines compared with GES1 (Fig. 1B). Collectively, these results suggest the upregulation of SMC4 in human CA.

\subsection{SMC4 knockout inhibits the proliferation of CA cells}


To investigate the SMC4 regulation effect on proliferation of CA cells, silenced control group cells (RNAiVector) and cells stably knocked out SMC4 (SMC4-RNAi1 and SMC4-RNAi2) of SGC-7901 and BGC-823 were constructed first. Then the expression level of SMC4 in SGC-7901 and BGC-823 cells stably transfected was identified by Western Blot and qRT-PCR respectively. The findings exhibited the significantly reduced expression level of SMC4 in the RNAi1 and RNAi2 groups as compared to the RNAiVector group (Fig. 2A-B). Then, we revealed the SMC4 effect on cell proliferation through MTT assay and observed that the SMC4 knockout significantly reduced the proliferation capacity of two strains of cells compared with the RNAi-Vector group (Fig. 2C). Consistent with this, cells knocked out of SMC4 showed a significantly reduced colony-forming capacity (Fig. 2D). In short, these results demonstrate that the proliferation of CA cells after SMC4 knockout is significantly reduced.

\subsection{SMC4 knockout can inhibit CA cells' invasion as well as migration}

To reveal the regulation effect of SMC4 on the migration of CA cells, we conducted a wound-healing experiment. The SMC4 knockout was found to inhibit the migration of BGC-823 and SGC-7901 cells (Fig. $3 A-B)$. At the same time, the transwell migration experiment can further confirm the above findings. Additionally, the transwell invasion assay showed that SMC4 knockout reduced cell invasion ability compared with the RNAi-Vector group (Fig. 3C-D). These outcomes indicate that SMC4 knockout can hinder the CA cell migration and invasion.

\subsection{SMC4 knockout can promote apoptosis and block the CA's cell cycle progression}

The effect of SMC4 knockout on cell apoptosis was subsequently investigated, and Annexin V/PI staining exhibited a significant rise in apoptosis rates in both lines after SMC4 knockdown than the RNAivector group. (Fig. 4A). The activation of apoptosis by SMC4 knockout was also validated by Western Blot analysis of apoptosis-related proteins. It was shown that knocking down SMC4 resulted in a drop in the anti-apoptotic protein $\mathrm{Bcl}-2$ with enhancement in the pro-apoptotic proteins Bax, caspase3, and Cleaved-caspase3 levels. (Fig. 4C). Simultaneously, we used PI labeling and flow cytometry to discover the influence of SMC4 deletion on cell cycle progression. In SMC4 knockout cells, a significant increase in G0/G1 phase cells was detected, with a reduced number of $S$ and G2/M phase cells, suggesting that SMC4 inhibition may induce G0/G1 phase arrest (Fig. 4B). Furthermore, Western Blot results revealed that when SMC4 was knocked out, the expression levels of proteins involved in cell cycle CDK4, CDK6, and Cyclin D1 were reduced, while the expression profiles of cell cycle inhibitor P21 were increased (Fig. 4D), confirming that SMC4 inhibition can cause cell cycle arrest in the G0/G1 phase. Therefore, we can find that SMC4 knockout can promote apoptosis of CA cells and induce G0/G1 phase arrest of cell cycle.

\subsection{SMC4 regulates the EMT process and PI3K/AKT signaling pathway of CA cells}


We used Western Blot to examine and assess the expression of the protein which is related to EMT in various cells following SMC4 deletion. The results showed that following SMC4 down-regulation, the expression of interstitial markers Vimentin and N-cadherin was dramatically reduced, but the expression of epithelial marker E-cadherin was dramatically elevated (Fig. 5A-B). These data suggest that SMC4 knockout inhibits epithelial-mesenchymal transformation. To learn more about SMC4's impact on CA cells, we examined that how it affects the proteins involved in the PI3K/AKT signaling pathway. We found that phosphorylated AKT, PI3K, and mTOR protein levels were significantly reduced in both CA cells after SMC4 knockout, while AKT, PI3K, and mTOR protein levels remained unchanged (Fig. 5C-D). This shows that the PI3K/AKT signaling pathway may be used by SMC4 to control the progression of CA. In conclusion, SMC4 knockout can inhibit the EMT process and inactivate the PI3K/ AKT signaling pathway.

\section{Discussion}

SMC4 expression was first observed in GES-1 and various CA cell lines MGC-803, SGC-7901, MKN-45, and BGC-823 in this investigation, and we discovered that SMC4 was significantly expressed in a range of CA cells. Then, we demonstrated the effect of two SMC4 interference knockout sequences in SGC-7901 and BGC-823 of two different CA cells. Furthermore, we discovered that knocking down SMC4 inhibited SGC7901 and BGC-823's ability to proliferate, migrate, and invade, promoted cell apoptosis, induced G0/G1 phase arrest of the cell cycle, inhibited the EMT process, and resulted in the inactivation of the PI3K/ AKT signaling pathway. Therefore, it can be suggested from these findings that SMC4 can promote the progression of CA.

SMC4 is a subunit of the protein-encoding "chromosomal structure maintenance" and is also necessary for promoting the development of cells from the G1 phase to the S phase [14]. In addition, SMC4 and SMC2 are the core components of the condensed protein complex, which are involved in the assembly and separation of chromosomes and are closely associated with the progression of the cell cycle and even the incidence and development of tumors [15]. Recent studies have shown that SMC4 overexpression can significantly promote proliferation, invasion, migration ability of glioma cells, and can mediate the invasive phenotype of glioma cells by activating TGF $\beta / S m a d$ signal [13]. Other research has found that knocking down SMC4 reduces hepatocellular carcinoma cell proliferation, and that SMC4 expression is linked to tumor size, advanced stage, dedifferentiation, and vascular invasion in primary hepatocellular carcinoma [10]. SMC4 deletion inhibits lung cancer cell growth and invasion, and acts as an independent prognostic factor [12]. SMC4 deletion impacts the ability of leukemia progression by lowering the fraction of leukemia stem cells [9]. In colorectal cancer, SMC4 knockout can also inhibit the invasion, migration, and proliferation, colorectal cancer cell cycle progression, and promote apoptosis [11]. However, the intrinsic relationship between SMC4 and CA remains unclear. We examined the regulatory relationship between SMC4 and CA in this study. Similarly, higher expression of SMC4 mRNA and protein were observed in CA cells than normal gastric epithelial cells, and down-regulation of SMC4 could significantly reduce cell proliferation, invasion, migration ability, promote cell apoptosis ability, and induce G0/G1 phase arrest of cell cycle. We believe that SMC4 can promote the tumorigenicity of CA cells and play an oncogene-like role in the occurrence and development of CA. 
EMT is a reversible biological process that temporarily changes epithelial cells into quasi mesenchymal cells. Epithelial cells gradually lose their pebble epithelial appearance and become fusiform mesenchymal cells during this phase [16]. Recent studies have shown that EMT actively participated in the incidence and progress of a variety of tumors, leading to highly invasive, drug-resistant, and stem-cell characteristics of cells, and easy formation of metastasis in distant organs, thus leading to cancer recurrence and metastasis [17]. It has been proved that some genes regulate the EMT process in CA and affect its progress [18], while whether SMC4 has a regulatory relationship with EMT in CA is not clear yet. Therefore, to further explore the role of SMC4 in CA, we studied the influence of knockdown of SMC4 on EMT-related proteins in CA. We found that down-regulation of SMC4 could significantly increase the epithelial marker E-cadherin expression, while the expression of interstitial marker $\mathrm{N}$-cadherin and Vimentin was significantly decreased. These findings suggest that SMC4 knockout can inhibit the process of epithelial-mesenchymal transformation of CA cells, and it can be further speculated that the down-regulation of SMC4 may have a certain blocking effect on the metastasis and recurrence of CA.

The PI3K/AKT signaling system, which begins with the activation of membrane receptor tyrosine kinases (RTKs), is one of the most critical signaling pathways in normal physiological functions [19]. The literatures revealed that the PI3K/ AKT signaling pathway is abnormally activated in different cancers, and the abnormal activation of this pathway is related to tumor growth, angiogenesis, and survival [2022]. Furthermore, certain genes have been found to influence the progression of $\mathrm{CA}$ by influencing the $\mathrm{PI} 3 \mathrm{~K} / \mathrm{AKT}$ signaling pathway [23]. Here, we discovered that this pathway is basically regulated by SMC4 in CA cells, i.e., knocking down SMC4 resulted in a decreased level of participating protein. As a result, downregulation of SMC4 in CA cells may result in the inactivation of the PI3K/AKT signaling pathway. In addition, it has been reported that abnormal activation of this pathway can regulate cell proliferation, metastasis, apoptosis, and autophagy, and it also exerts a core role in the regulation of epithelialmesenchymal transformation and chemotherapy resistance [24]. Therefore, the inhibition of SMC4 knockout on proliferation, migration, invasion ability, cycle progression, and EMT process of CA cells and the promotion of cell apoptosis may be achieved via regulation of PI3K/ AKT signaling pathway. The internal regulation mechanism of SMC4 on PI3K/ AKT in CA cells still needs to be further studied.

\section{Conclusions}

In conclusion, these findings imply that knocking out SMC4 has an inhibitory effect on the onset and progression of CA, which could be achieved by inactivating the PI3K/AKT signaling pathway. This suggests that SMC4 may be a potential prognostic and therapeutic target for CA.

\section{Abbreviations}

SMC4: Structural maintenance of chromosome protein 4

CA: Cardia adenocarcinoma 
EMT: epithelial-mesenchymal transition

GC: gastric cancer

\section{Declarations}

\section{Ethics approval and consent to participate}

Not required.

\section{consent for publication}

Agreement.

\section{Availability of data and material}

All data generated or analysed during this study are included in this published article.

\section{Competing interests}

The authors declare that they have no known competing financial interests or personal relationships that could have appeared to influence the work reported in this paper.

\section{Funding}

This study was supported by 512 Talent Cultivation Program (by51202207) and Postgraduate Research Innovation Program of Bengbu Medical College (Byycx20042).

\section{Authors' contributions}

Zhu Mengqi: Conceptualization, Methodology, Software, Investigation, Writing - original draft. Zhang Xinxin: Validation, Formal analysis, Visualization, Software. Gao Kaiji: Validation, Formal analysis, Visualization. Zhang Lingmei: Resources, Writing - review \& editing, Supervision, Data curation. Feng Xiaojia: Resources, Writing - review \& editing, Supervision, Data curation. Wang Hui: Resources, Writing review \& editing, Supervision.Jianguang Jia: Writing - review \& editing.

\section{Acknowledgments}

We would like to express our gratitude to the Anhui Biochemical and Pharmaceutical Engineering Research Center of Bengbu Medical College for their help and our sincere thanks to Mr. Liu Hao's research team.

\section{References}


1. Smyth EC, Nilsson M, Grabsch HI, van Grieken NC, Lordick F. Gastric cancer. Lancet. 2020. 396(10251): 635-648.

2. Chen W, Zheng R, Baade PD, et al. Cancer statistics in China, 2015. CA Cancer J Clin. 2016. 66(2): 115-32.

3. Olefson S, Moss SF. Obesity and related risk factors in gastric cardia adenocarcinoma. Gastric Cancer. 2015. 18(1): 23-32.

4. Jia J, Zhang X, Zhan D, et al. LncRNA H19 interacted with miR-130a-3p and miR-17-5p to modify radio-resistance and chemo-sensitivity of cardiac carcinoma cells. Cancer Med. 2019. 8(4): 16041618.

5. Xu H, Zhang L, Miao J, et al. Patterns of recurrence in adenocarcinoma of the esophagogastric junction: a retrospective study. World J Surg Oncol. 2020. 18(1): 144.

6. Cao J, Yang T, Wang G, et al. Analysis of the clinicopathological features and prognostic factors in 734 cases of Chinese Hui and Han patients with adenocarcinoma of the esophagogastric junction. Surg Oncol. 2018. 27(3): 556-562.

7. Ajani JA, Amico TA, Bentrem DJ, et al. Esophageal and Esophagogastric Junction Cancers, Version 2.2019, NCCN Clinical Practice Guidelines in Oncology. J Natl Compr Canc Netw. 2019. 17(7): 855883.

8. Ma RM, Yang F, Huang DP, Zheng M, Wang YL. The Prognostic Value of the Expression of SMC4 mRNA in Breast Cancer. Dis Markers. 2019. 2019: 2183057.

9. Peng $L$, Tang $Y$, Zhang $Y$, et al. Structural maintenance of chromosomes 4 is required for leukemia stem cell maintenance in MLL-AF9 induced acute myeloid leukemia. Leuk Lymphoma. 2018. 59(10): 2423-2430.

10. Zhou B, Yuan T, Liu M, et al. Overexpression of the structural maintenance of chromosome 4 protein is associated with tumor de-differentiation, advanced stage and vascular invasion of primary liver cancer. Oncol Rep. 2012. 28(4): 1263-8.

11. Feng XD, Song Q, Li CW, et al. Structural maintenance of chromosomes 4 is a predictor of survival and a novel therapeutic target in colorectal cancer. Asian Pac J Cancer Prev. 2014. 15(21): 9459-65.

12. Zhang C, Kuang M, Li M, Feng L, Zhang K, Cheng S. SMC4, which is essentially involved in lung development, is associated with lung adenocarcinoma progression. Sci Rep. 2016. 6: 34508.

13. Jiang L, Zhou J, Zhong D, et al. Overexpression of SMC4 activates TGF $\beta /$ Smad signaling and promotes aggressive phenotype in glioma cells. Oncogenesis. 2017. 6(3): e301.

14. Chen Y, Huang F, Deng L, et al. HIF-1-miR-219-SMC4 Regulatory Pathway Promoting Proliferation and Migration of HCC under Hypoxic Condition. Biomed Res Int. 2019. 2019: 8983704.

15. Zhou J, Wu G, Tong Z, et al. Prognostic relevance of SMC family gene expression in human sarcoma. Aging (Albany NY). 2020. 13(1): 1473-1487.

16. Dongre A, Weinberg RA. New insights into the mechanisms of epithelial-mesenchymal transition and implications for cancer. Nat Rev Mol Cell Biol. 2019. 20(2): 69-84. 
17. Zhang Y, Weinberg RA. Epithelial-to-mesenchymal transition in cancer: complexity and opportunities. Front Med. 2018. 12(4): 361-373.

18. Zhang $X$, Zhu $M$, Wang $H$, et al. Overexpression of NCAPG inhibits cardia adenocarcinoma apoptosis and promotes epithelial-mesenchymal transition through the Wnt/ $\beta$-catenin signaling pathway. Gene. 2021. 766: 145163.

19. Yang Q, Jiang W, Hou P. Emerging role of PI3K/AKT in tumor-related epigenetic regulation. Semin Cancer Biol. 2019. 59: 112-124.

20. Chen H, Zhou L, Wu X, et al. The PI3K/AKT pathway in the pathogenesis of prostate cancer. Front Biosci (Landmark Ed). 2016. 21: 1084-91.

21. Ediriweera MK, Tennekoon KH, Samarakoon SR. Role of the PI3K/AKT/mTOR signaling pathway in ovarian cancer: Biological and therapeutic significance. Semin Cancer Biol. 2019. 59: 147-160.

22. Noorolyai S, Shajari N, Baghbani E, Sadreddini S, Baradaran B. The relation between PI3K/AKT signalling pathway and cancer. Gene. 2019. 698: 120-128.

23. Zhang $X$, Wang $H$, Han $Y$, et al. NCAPG Induces Cell Proliferation in Cardia Adenocarcinoma via PI3K/AKT Signaling Pathway. Onco Targets Ther. 2020. 13: 11315-11326.

24. Fattahi S, Amjadi-Moheb F, Tabaripour R, Ashrafi GH, Akhavan-Niaki H. PI3K/AKT/mTOR signaling in gastric cancer: Epigenetics and beyond. Life Sci. 2020. 262: 118513.

\section{Figures}

A
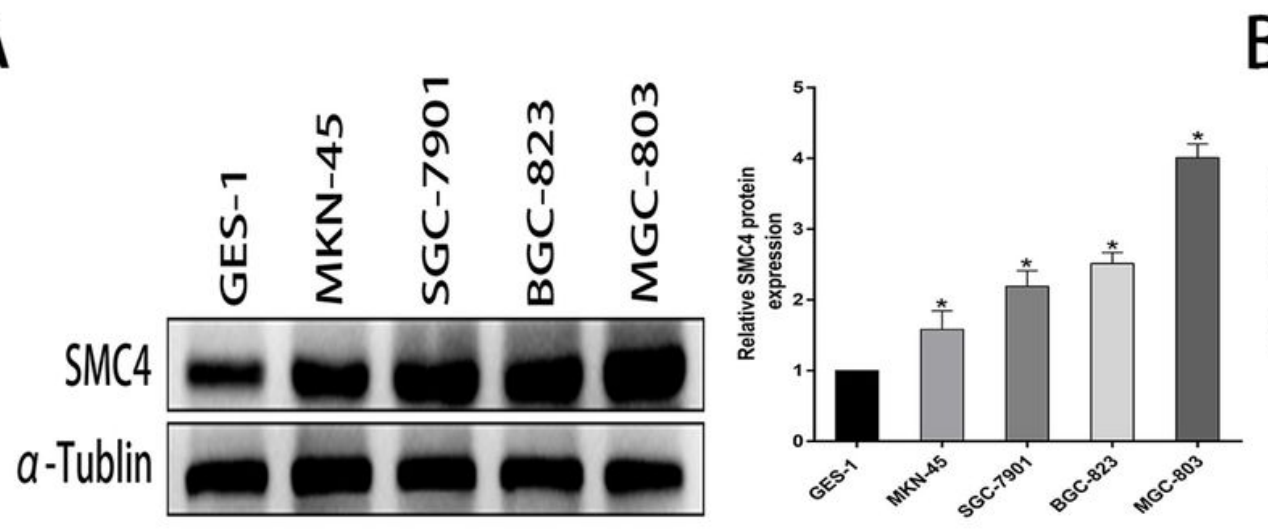

B

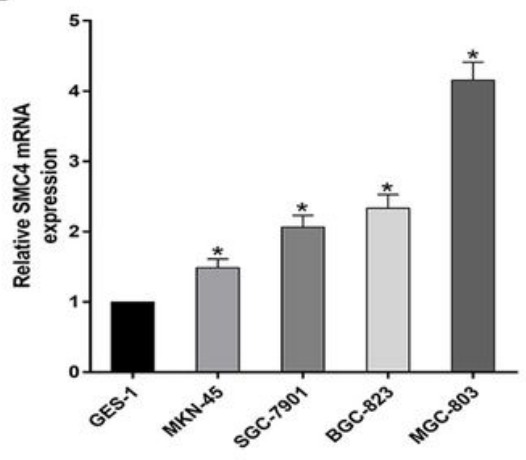

\section{Figure 1}

SMC4 was highly expressed in CA.A. Western Blot was utilized to evaluate the level of SMC4 protein expression in CA cells. B. qRT-PCR was used to evaluate the SMC4 expression in CA cells. 
A

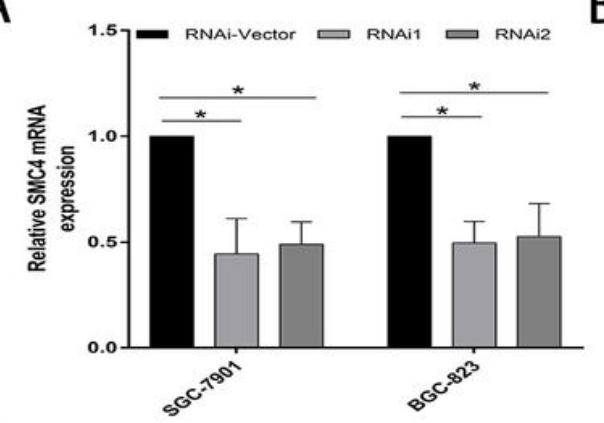

C

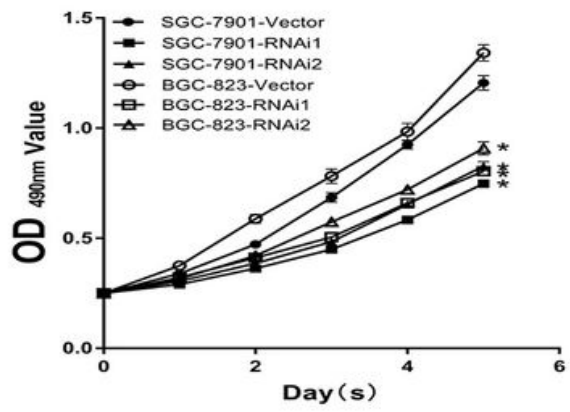

B
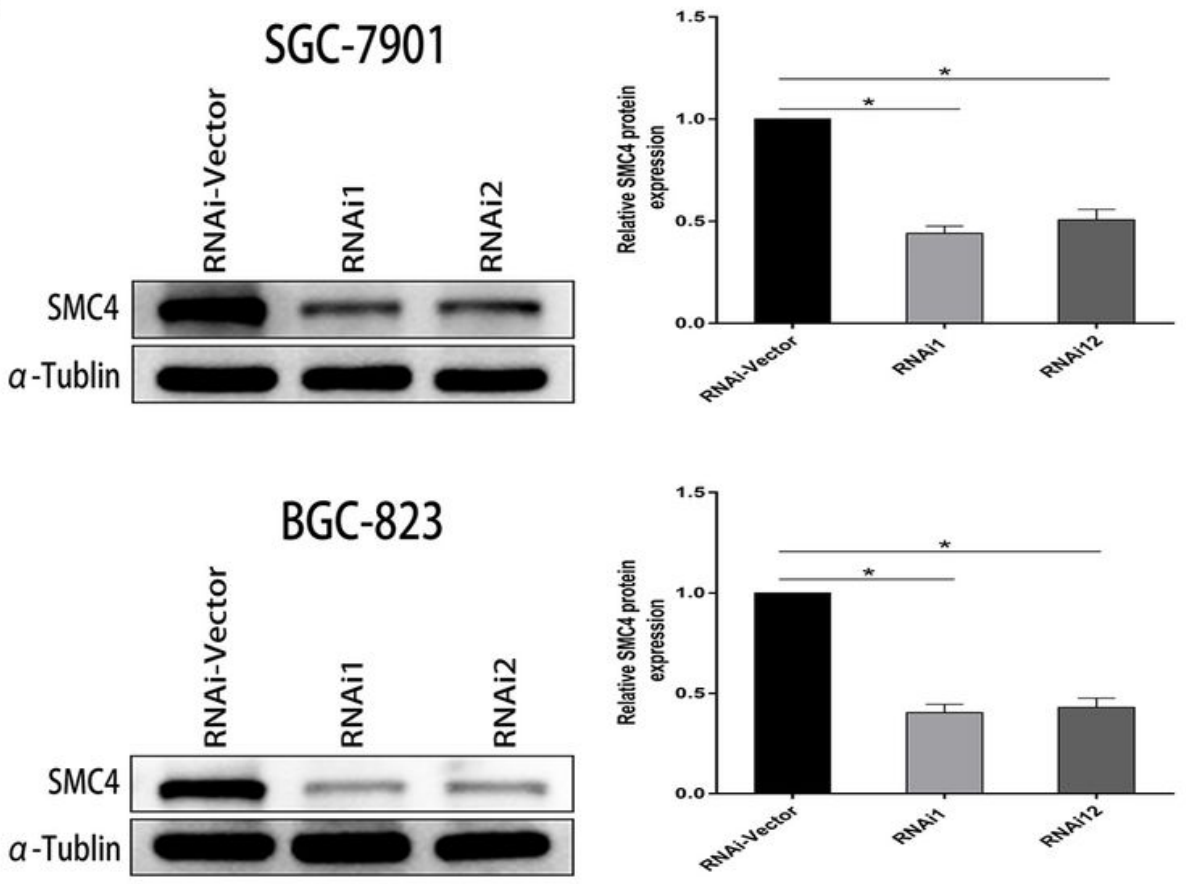

D
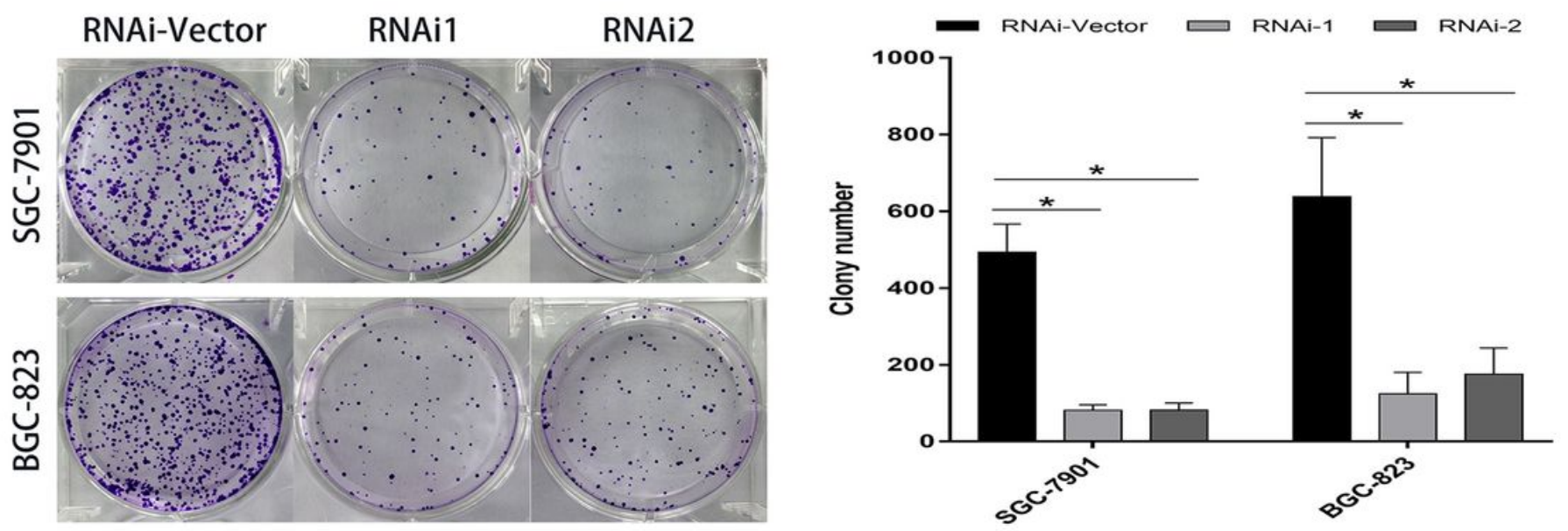

Figure 2

SMC4 knockout inhibited the CA cells proliferation. A. The expression of SMC4 in different CA cells transfected with RNAi-Vector, SMC4-RNAi1, and SMC4-RNAi2 was analyzed by the qRT-PCR assay. B. The expression level of SMC4 in two kinds of CA cells transfected with RNAi-Vector, SMC4-RNAi1, and SMC4RNAi2 was analyzed by Western Blot. C. According to the MTT assay, SMC4 knockout significantly reduced the cell proliferation capability. D. SMC4 knockout reduced the average number of colonies in the clone formation test. 
A
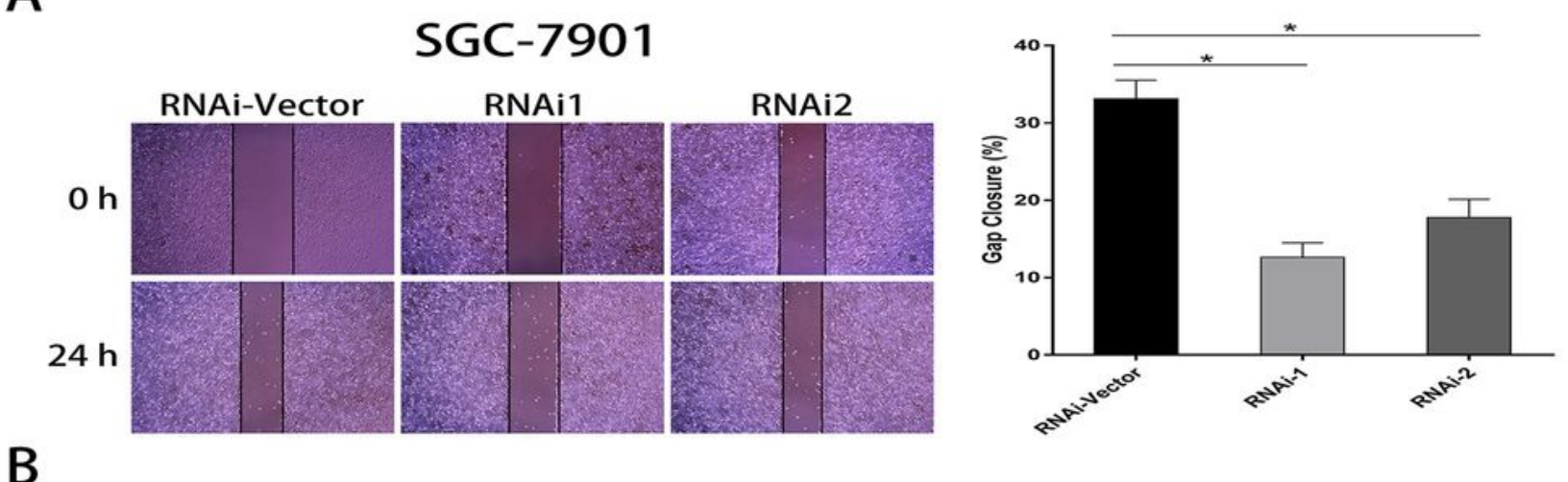

B

\section{BGC-823}
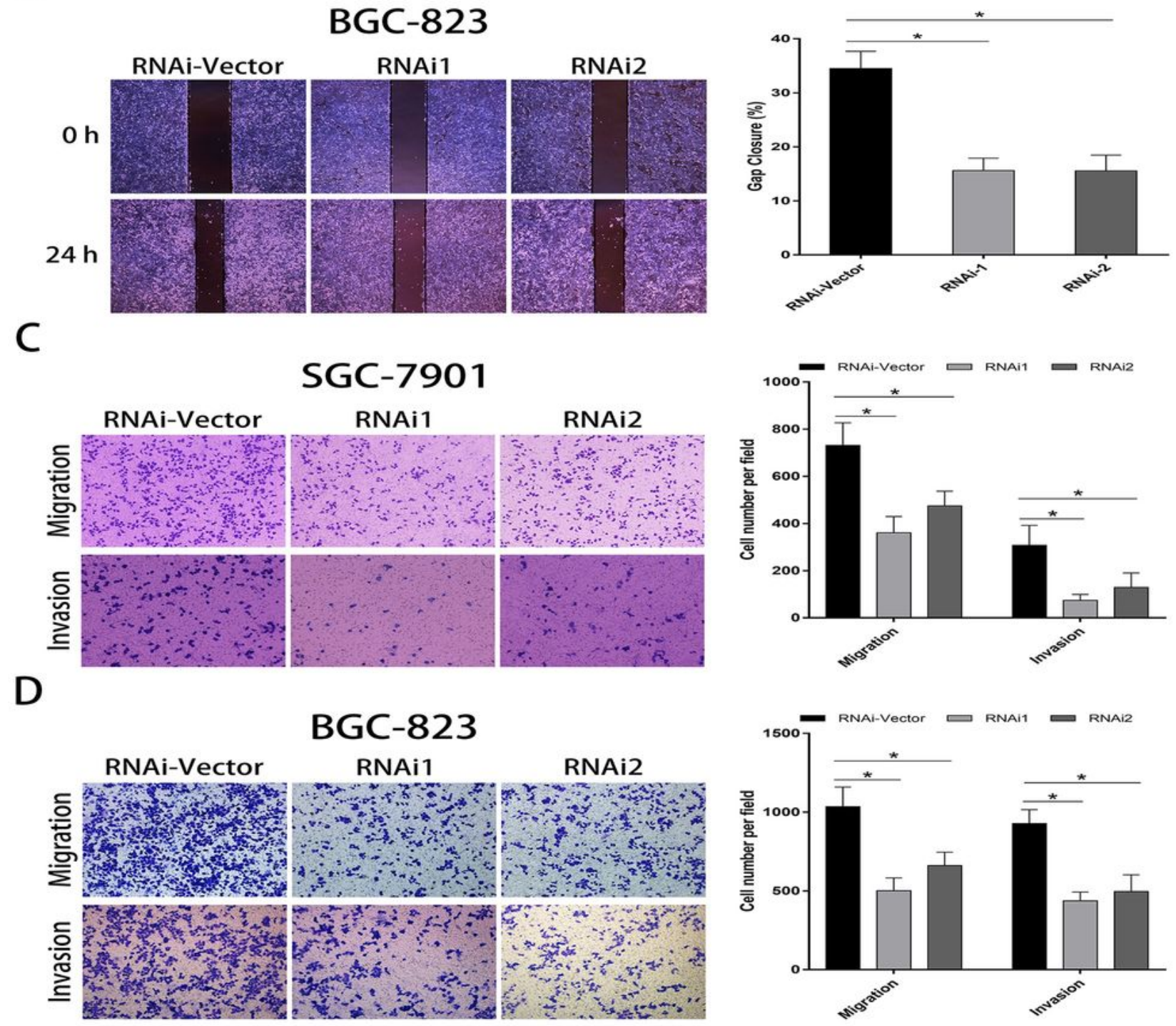

Figure 3

CA cells migrated and invaded less when SMC4 was knocked off. A. wound-healing experiment looked at the effect of SMC4 deletion on the migration of the CA cell line SGC-7901. B. The effect of SMC4 knockout on the migration of CA cell BGC-823 was studied in the wound healing experiment. C. The effect of SMC4 knockout on the invasion and the migration capability of SGC-7901 was evaluated by the 
Transwell experiment. D. The effect of SMC4 deletion on the invasion and migrating capabilities of CA cell line BGC-823 was studied using a transwell experiment.

A

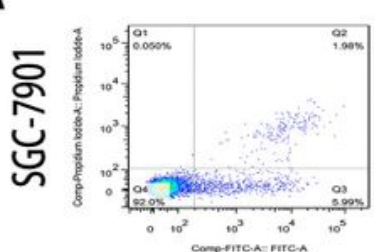

RNAi-Vector

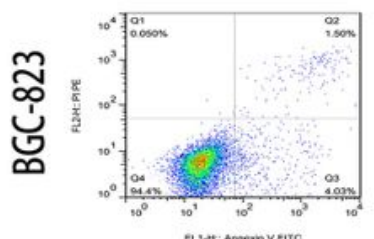

RNAi-Vector

B

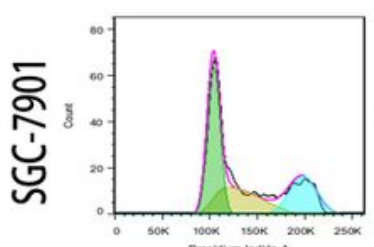

RNAi-Vector

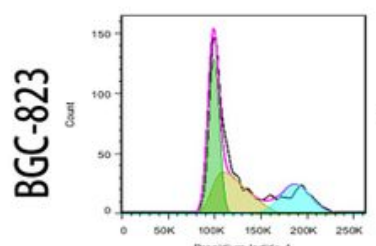

RNAi-Vector

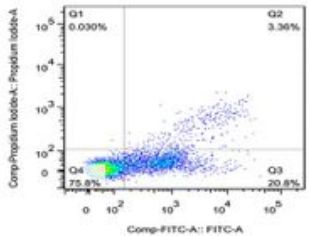

RNAi1

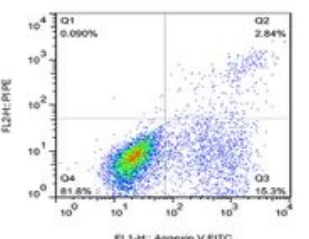

RNAi1

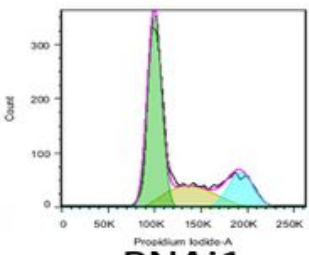

RNAi1

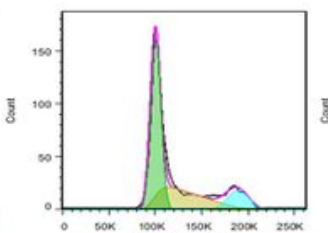

RNAi1

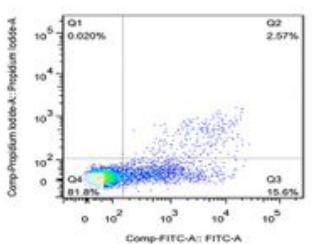

RNAi2

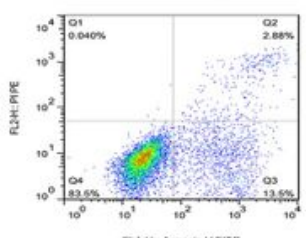

RNAi2

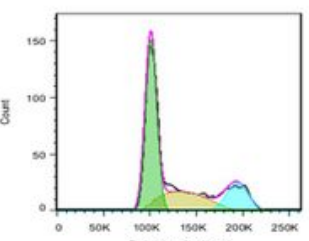

RNAi2

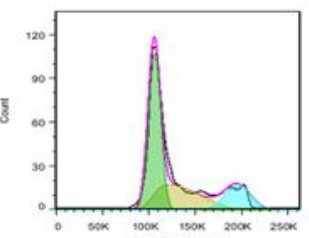

$\mathrm{RNAi} 2$
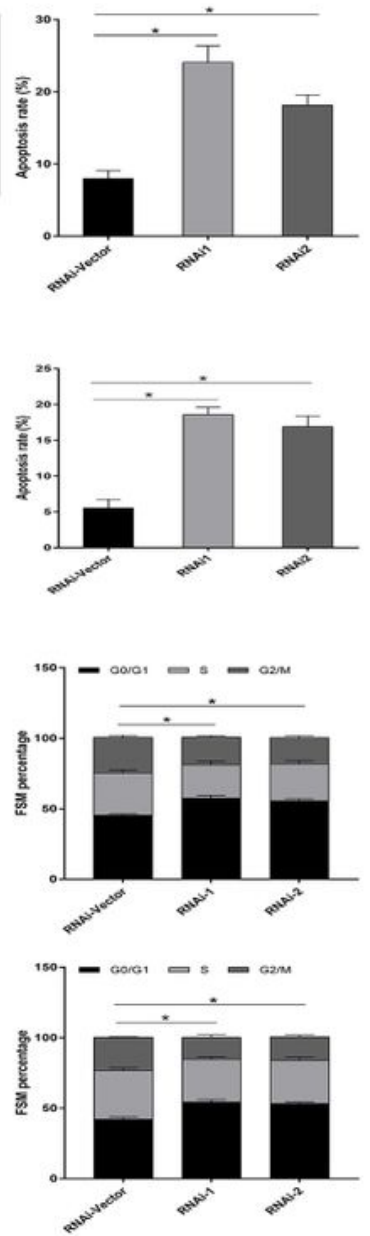

C
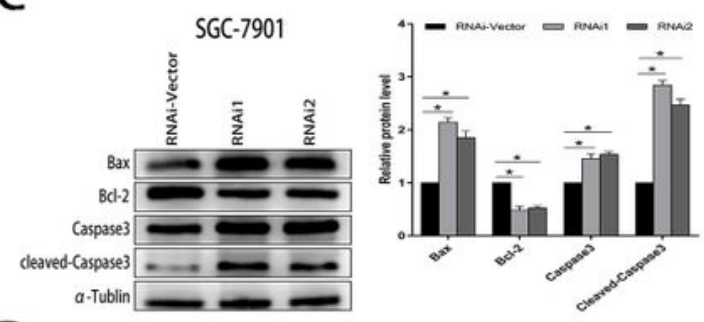

D
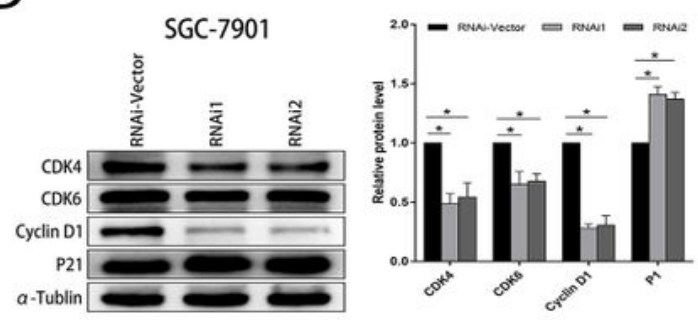
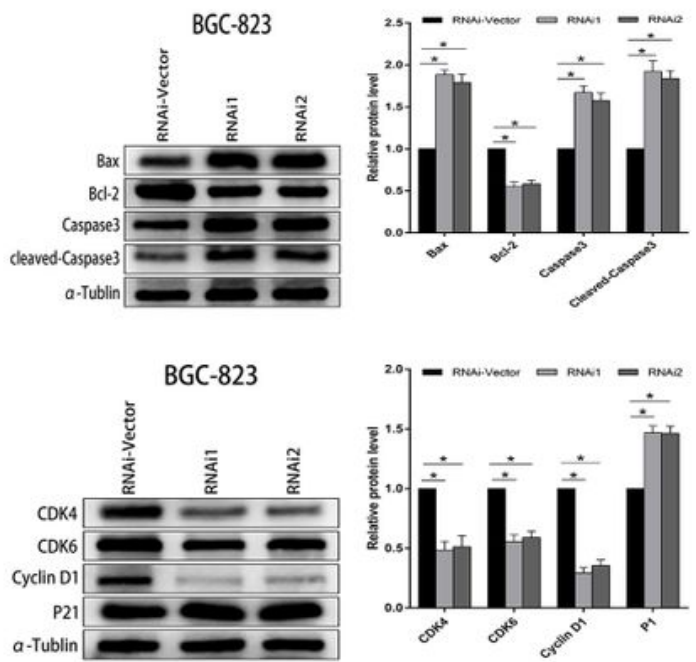

Figure 4

SMC4 knockout can promote apoptosis of CA cells and hinder cell cycle progression. A. A representative summary bar (right) and image (left) of the effect of SMC4 knockout on apoptosis in two CA cells from the flow cytometry Annexin V-FITC / PI sting experiment. B. A representative image (left) and a summary 
histogram (right) of the effect of stripping SMC4 on the cell cycle of different CA were shown by flow cytometry PI training. C. Expression of proteins involved in apoptosis Bcl-2, Bax, Cleaved- caspase3 and caspase3 after SMC4 knockout. D. Expression of cyclin-related proteins CDK6, CDK4, Cyclin D1, and P21 after SMC4 knockout.

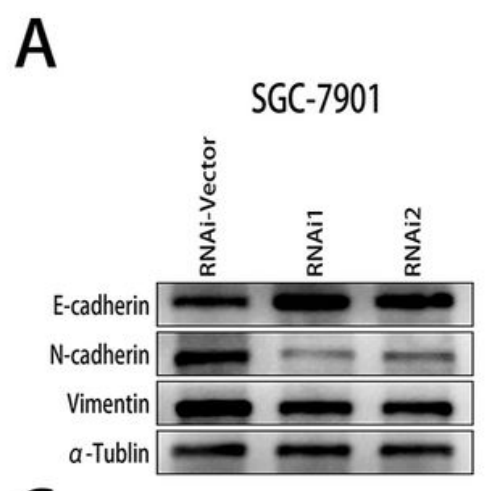

C

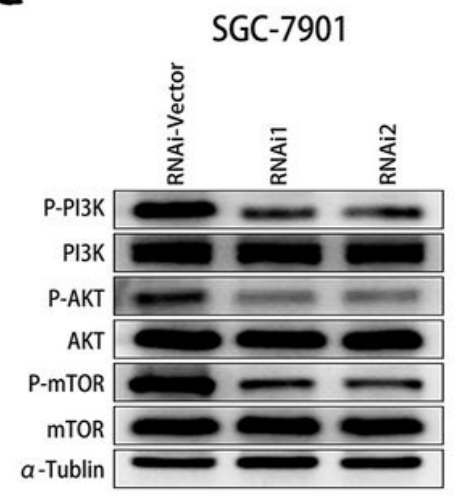

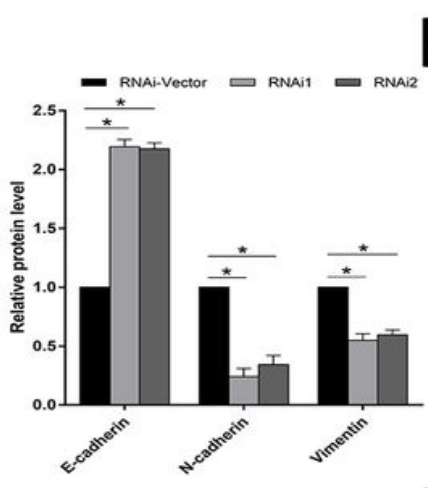

B
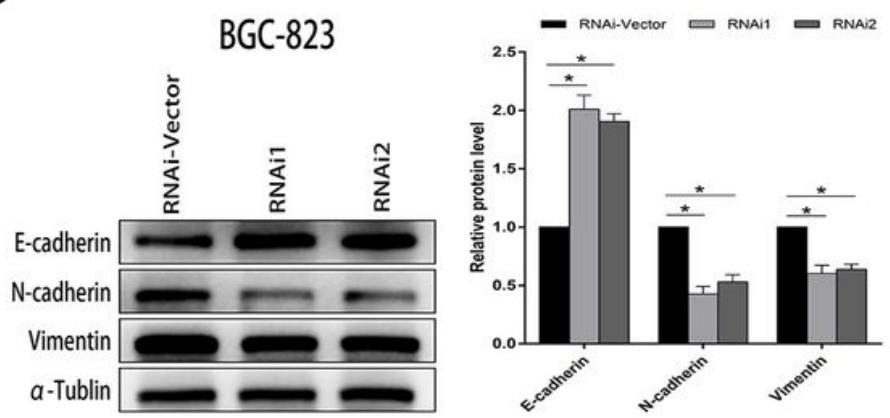

D

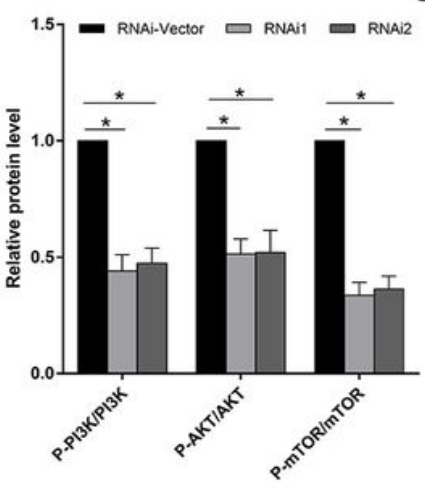

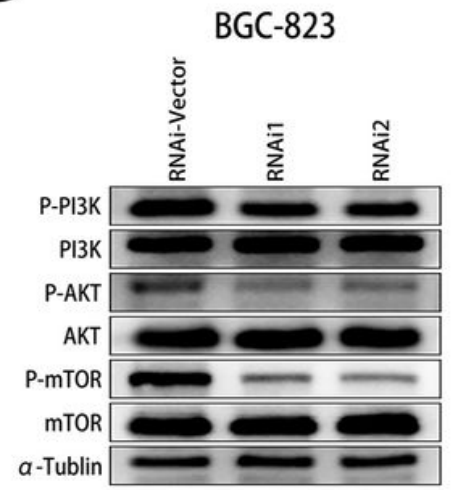

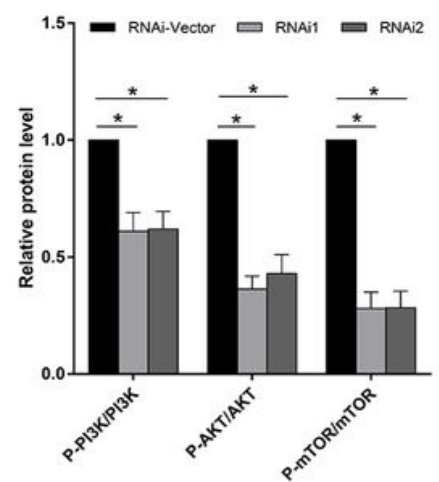

\section{Figure 5}

SMC4 regulates the EMT process and PI3K/ AKT signaling pathway of CA cells. A-B. Expression of EMTrelated markers N-cadherin, E-cadherin, and Vimentin after SMC4 knockout in SGC-7901 cells and BGC823 cells. C-D. The expressions of PI3K/ AKT signaling pathway-related proteins PI3K, AKT, P-PI3K, P-AKT, P-mTOR, and mTOR in SGC-7901 and BGC-823 were evaluated by Western Blot after SMC4 knockout. 